\title{
Study of the Discrete Wavelet Transform based Designed System under different simulation conditions
}

\author{
Simmi Garg \\ Department of Physics \\ ${ }^{1}$ I K G Punjab Technical University \\ Kapurthala-Jalandhar Highway, Kapurthala \\ ${ }^{2}$ Hans Raj Mahila Maha Vidyalaya, Jalandhar \\ India \\ Anuj Kumar Sharma \\ Department of Mathematics \\ L R DAV College \\ Jagraon, Punjab \\ India \\ Anand Kumar Tyagi \\ Department of Applied Physics \\ S B S State Technical Campus \\ Ferozepur, Punjab \\ India
}

Received: November 17, 2020, Revised: December 17, 2020, Accepted: December 21, 2020.

Published: December 21, 2020.

\begin{abstract}
Wavelet Transforms is an Important Part of, Systems Theory and Signal Processing and finds numerous important applications in Science and Engineering. In this paper, we investigated the performance of proposed scheme coded Discrete wavelet transform based Orthogonal frequency division multiplexing scheme over Additive white Gaussian noise channel using Pulse Amplitude Modulation in terms of Energy bits per noise ratio values. The simulation has been done using MATLAB software and results are compared with $1 / 2$ rate convolution coded Discrete wavelet transform based Orthogonal frequency division multiplexing system. It is found by MATLAB simulations that the performance of proposed scheme coded Discrete wavelet transform based Orthogonal frequency division multiplexing outperforms than that of $1 / 2$ rate convolution encoded Discrete wavelet transform based Orthogonal frequency division multiplexing with 16-Pulse Amplitude Modulation. Along with this, different orders of reverse biorthogonal and biorthogonal wavelets are implemented
\end{abstract}

to simulate the proposed system with 16-Pulse Amplitude Modulation scheme. The performance of proposed system is compared and it is found that proposed system performs better than conventional system under all different simulation conditions. This study finds important applications in Signal Processing.

Keywords- Signals, Signal Processing, Systems Theory, Systems Engineering, Wavelets, OFDM, DWT, Convolution codes, PAM,.

\section{INTRODUCTION}

Wavelet Transforms is an Important Part of, Systems Theory and Signal Processing and finds numerous important applications in Science and Engineering. In the last decades, multicarrier communication based on orthogonal frequency division multiplexing (OFDM) [1-3] has

emerged as one of major techniques for wireless communication systems and signal processing. In this scheme data is transmitted on number of parallel sub carriers. But due to various channel impairments, the orthogonality between the sub carriers gets distorted. In order to improve the 
orthogonality between the sub carriers, wavelet based OFDM [4] came in to picture. In Wavelet based OFDM, signal is localized in both time and frequency domain. Wavelet based OFDM provide better reconstruction of the original signal as compare to conventional OFDM. Since, wavelets maintain their orthogonality even in difficult channel conditions [5], so wavelet based OFDM system provide more robustness against the inter symbol interference (ISI) and inter carrier interference (ICI). Further, DWT-OFDM has much higher spectrum efficiency than conventional OFDM. Channel coding has been proved to provide improvement in reliability of communication system. Quality of communication can be improved by using error control schemes. The error detection and correction scheme [6] is mainly classified in to automatic repeat request (ARQ) and forward error correction (FEC) scheme. ARQ [7] require resending of the corrupted data bits while FEC provides the ability of detecting and correcting the error bits without he requirement of resending the data bits. So, FEC is more significant scheme when the resending of data bits is either not possible or becomes costly. It can connect one sender with number of users like in broad cast messages. In FEC [8],[9], a number of redundant bits are sent along with the original data bits for correcting the error bits. Convolution codes are most widely used in error detection and correction area. Convolution codes consist of shift register and modulo2 adder. Constraint length of the encoder denotes the number of number of shift registers used for realization of that encoder. Code rate of the convolution code is the ratio of number of input bits and number of output bits of the encoder. Coding leads to increase in the performance of the communication system and decrease the bit error rate (BER) [10], [11]. Due to its ability to improve the performance of the communication system, the data bits are first coded using a suitable encoder and then sent through the communication channel [12],[13].

Fazel k. et al.[14] has studied the performance of convolution encoded OFDM system. It is shown in the results that coding leads to decrease in bit error rate and improves the performance of the communication system. Rashed. et al.[15] studied the performance of convolution coded OFDM and cyclic redundancy code coded OFDM system over AWGN channel and fading (Rayleigh and Rician) channels. QPSK modulation has been used to implement the system. Is found by computer simulations that Convolution interleaved based OFDM system outperform the cyclic reduncy coded interleaved OFDM as well as uncoded OFDM system. Kafaltiya et al. [16] studied the effect of different modulation schemes on the performance of OFDM system over AWGN channel. Different orders of QAM and PSK modulations schemes are used for modulating the data. It is shown in the results that BER is minimum for 16-QAM modulation scheme. Kansal et al. [17] proposed an OFDM based MPSK wireless system to reduce BER will sending the data through Rayleigh fading channel. Results show that simulating system using BCH code reduces the BER than using Reed solomon codes. Hader et al. [18] implemented convolution coded OFDM system using QAM and QPSK modulation over AWGN, Rayleigh and Racian fading channel. It is shown in the paper that convolution coded OFDM system performs best with
QAM modulation scheme over all different types of communication channel. Pooja et al. [19] propose a matrix interleaver for coded OFDM system. In the paper four FEC schemes have been designed in order to reduce bit error rate. Relative comparisons between different designed FEC schemes have been studied. It is found in the results that single convolution coded and dual interleaving scheme performs better out of all. Sharma et al. [20] studied the BER performance of conventional and wavelet based OFDM over AWGN channel. S Garg et al. [21] proposed an error correction scheme for correcting consecutive errors and the results depicted that the proposed scheme outperforms the convolution scheme in low Eb/No range.

In this paper, performance of DWT-OFDM of scheme proposed in [21] is examined using PAM modulation and haar, different members of biorthogonal wavelet family and different members of reverse orthogonal wavelet family over AWGN channel for wireless communication scenario. The main objective of this study is to propose an error correction scheme that can perform better than present $1 / 2$ rate convolution coded system and to improve the performance of today's communication system and compare the results with $1 / 2$ rate convolution coded DWT-OFDM. This study finds important applications in Signal Processing [22], [23], [24], [25].

\section{PROPOSED SCHEME}

Proposed scheme provide the guard against two bit errors occurring during transmission of the data bits through communication channel. Proposed encoder consists of two shift registers and one modulo2 adder. Shift registers are used for temporary storage of data bits. Modulo 2 adder is basically a EXOR logic circuit which generate coded bit 1 only if one of the two bits in the registers is 1 otherwise the output bit will be 0 . Proposed encoder is of $1 / 2$ rate i.e. for each 1 input bit, output of the encoder consists of 2 bits just like a $1 / 2$ rate convolution encoder.

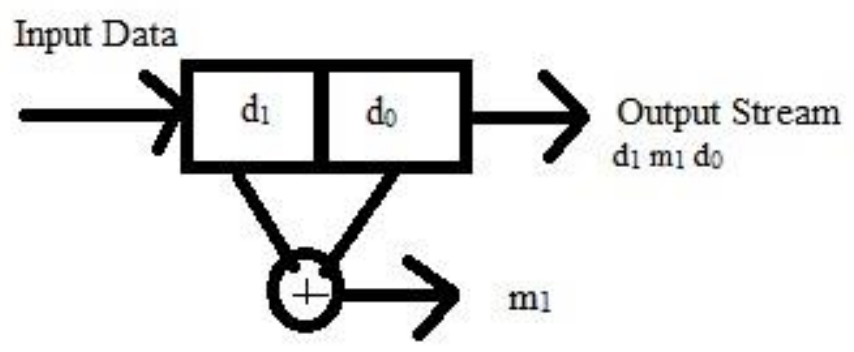

Figure 1 Diagram of the proposed encoder.

Here, $d_{0}$ and $d_{1}$ represents the first and second bit of the input data stream. $\mathrm{m}_{1}$ represents the output of the modulo 2 adder. Initially, both shift registers hold binary 0 's. The input bits are inserted in the shift register so that there are 2 bits held in the two shift register. Then, EXOR gate performs its function. After the EXOR gate operation, the bits in the shift registers are shifted towards the right. So that the bit in the rightmost shift register is ejected out of the registers. The output stream 
consists of the bit in the rightmost register followed by the output of the modulo 2 adder. Then the process is repeated with second bit in the rightmost register and third input bit in the left register. This process is repeated along the whole length of the input data stream. At the end of the encoding process, the encoded data bits consists of the input data bits at the odd places and their corresponding EXOR gate outputs at even places. The proposed scheme is very efficient and provides more robustness against the error occurring during transmission of the data bits.

The proposed scheme has a wide scope and can be implemented to code DWT-OFDM system. The proposed scheme does not require retransmission of the corrupted data bits which is one of the major advantages of the scheme. Further, BER reduces to a great extent when proposed scheme is implemented to DWT-OFDM system. This work is done to investigate the performance of the proposed scheme coded DWT-OFDM system using 16-PAM modulation scheme over AWGN channel. It is found that proposed system outperforms the conventional $1 / 2$ rate convolution encoded DWT-OFDM system.

\section{SIMULTION MODEL}

The main objective of our work is to simulate the proposed scheme coded DWT-OFDM using wavelet family and PAM modulation scheme. The block diagram of the proposed system is shown in the figure 2 .

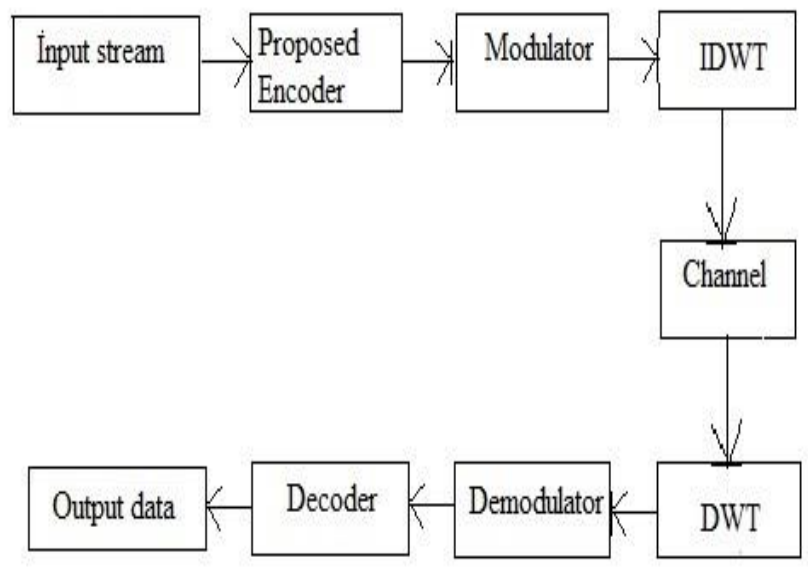

Figure 2 Block diagram of simulation model

For simulating the desired communication system, we have considered randomly generated input data bits. The number of input data bits is 64000. MATLAB software is used to write algorithm for the proposed scheme coded DWT-OFDM system. Various parameters are changed to study the performance of proposed system.

Table1. Parameters used for simulation of the system

\begin{tabular}{|l|l|l|l|}
\hline $\begin{array}{l}\text { Sr. } \\
\text { No. }\end{array}$ & Parameter & $\begin{array}{l}\text { Proposed } \\
\text { scheme coded } \\
\text { DWT-OFDM }\end{array}$ & $\begin{array}{l}\text { Convolution } \\
\text { encoded } \\
\text { DWT-OFDM }\end{array}$ \\
\hline 1. & Modulation & 16-PAM & 16-PAM \\
\hline 2. & $\begin{array}{l}\text { Error correction } \\
\text { code }\end{array}$ & $\begin{array}{l}\text { Proposed } \\
\text { scheme }\end{array}$ & $\begin{array}{l}\text { Convolution } \\
\text { scheme }\end{array}$ \\
\hline
\end{tabular}

\begin{tabular}{|l|l|l|l|}
\hline 3. & Code Rate & $1 / 2$ & $1 / 2$ \\
\hline 4. & Eb/No Range & 1 to $15 \mathrm{Db}$ & 1 to $15 \mathrm{~dB}$ \\
\hline 5. & $\begin{array}{l}\text { Communication } \\
\text { Channel }\end{array}$ & AWGN & AWGN \\
\hline 6. & Data Length & 64000 & 64000 \\
\hline
\end{tabular}

The algorithm of the proposed system is as follows:

1. Input data stream is generated using random integer 0 and 1.

2. Encoding of the input data streams with the proposed encoder / convolution encoder has been done to simulate two systems.

3. Modulation of the encoded data is done using 16-PAM modulation scheme.

4. Perform serial to parallel conversion

5. Discrete wavelet transforms of the modulated data bits is done. Haar, different members of biorthogonal wavelet family and different members of reverse biorthogonal wavelet family are used for performing DWT function.

6. Noise is inserted in the system by transmitting the data bits through AWGN channel.

7. At the receiver side, reverse operations are performed to retrieve the received data bits from the received bits.

8. Decoded bits are compared with the original data bits to find the incorporated bits.

9. BER as a function of Eb/No values are plotted.

\section{RESULTS AND DISCUSSION}

In our study, we have done simulations to study the performance of proposed scheme coded DWT-OFDM system. 16-PAM modulation scheme has been used to simulate the proposed system. Different parameters used for simulations have been defined in section4. The BER plots of the proposed system have been compared with that of $1 / 2$ rate convolution coded DWT-OFDM system over AWGN channel. Figure 3 represents the performance of proposed scheme coded DWTOFDM and $1 / 2$ rate convolution coded DWT-OFDM using Haar wavelet and 16-PAM modulation. It is clear from the graph that proposed scheme coded DWT-OFDM outperforms $1 / 2$ rate convolution coded DWT-OFDM system. 


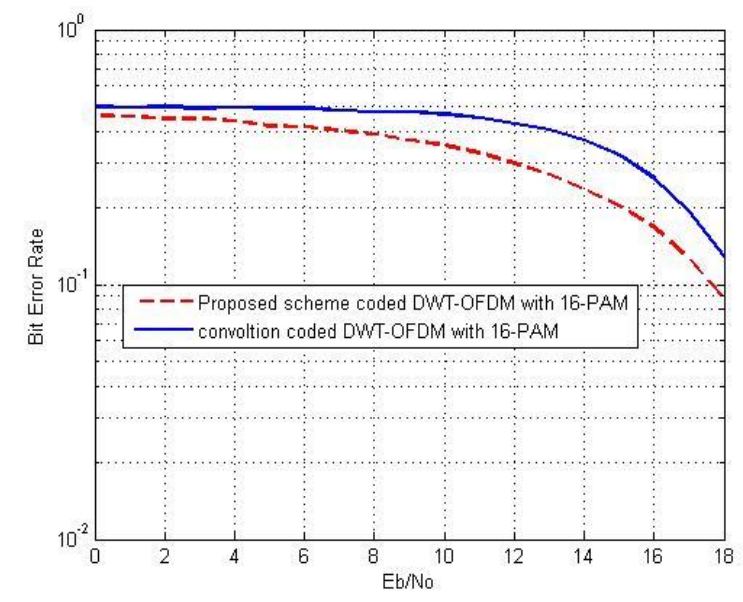

Figure 3 Performance of proposed scheme coded DWTOFDM system using 16-PAM modulation

Fig.4 represents the performance of proposed scheme coded DWT-OFDM system and $1 / 2$ rate convolution encoded DWTOFDM system with haar and biorthogonal5.5 wavelet using 16-PAM modulation scheme. It is seen in the results that proposed system can give performance improvement over $1 / 2$ rate convolution coded DWT-OFDM system with both haar and biorthogonal5.5 wavelet. The performance of the proposed system is best when biorthogonal5.5 wavelet is used with 16PAM modulation scheme.

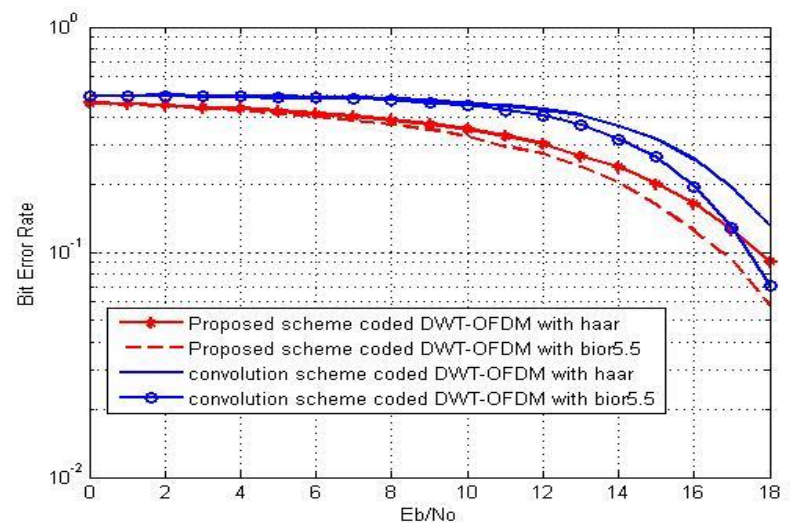

Figure 4 Performance of proposed system with haar and biorthogonal5.5 wavelet using 16-PAM modulation

We have also investigated the performance comparison of the proposed scheme coded DWT-OFDM system using different members of biorthogonal wavelet family over AWGN channel and 16-PAM modulation scheme in figure 5. It is investigated that BER of proposed system decreases as the order of biorthogonal wavelet increases. In other words, proposed system performs best with biorthogonal5.5 wavelet followed by biorthogonal4.4 and then with biorthogonal 3.1 wavelet. Hence, it can be concluded that as the order of wavelet increases, the BER of the system decreases.

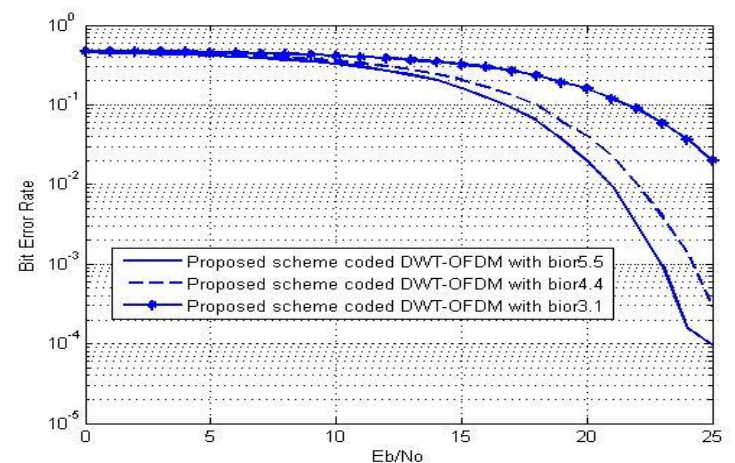

Figure 5 Performance comparison of proposed system using different members of biorthogonal wavelet family

The performance comparison between proposed system and $1 / 2$ rate convolution coded DWT-OFDM using haar and reverse biorthogonal5.5 wavelet over AWGN channel and 16-PAM modulation scheme is represented in figure6. It is seen from the results that proposed system outperform the conventional coded system with both types of wavelets. BER values for proposed scheme coded DWT-OFDM are higher with reverse biorthogonal5.5 wavelet than with haar wavelet. Figure 7 depict the performance of proposed scheme coded DWTOFDM using different members of reverse biorthogonal wavelet family with 16-PAM modulation over AWGN channel. Proposed system performs best with reverse biorthogonal 3.3 wavelet.

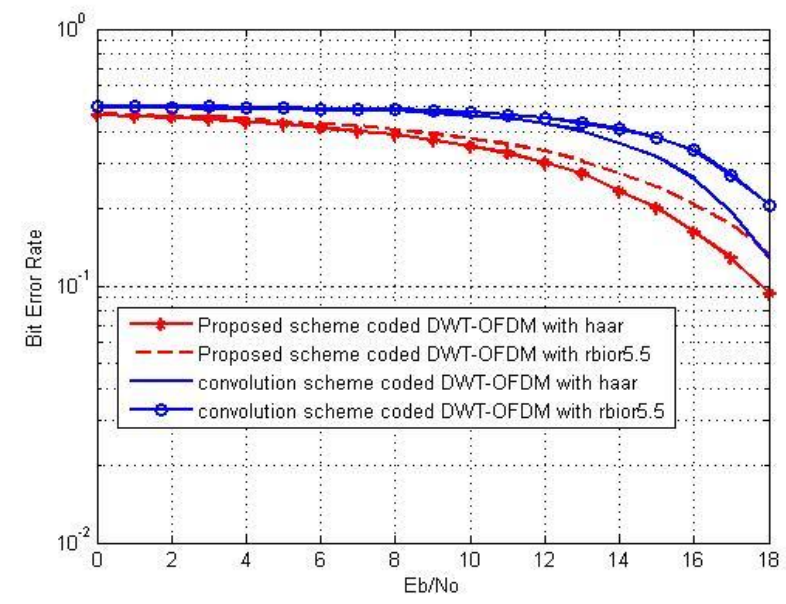

Figure 6 Performance of proposed system with haar and reverse biorthogonal5.5 wavelet using 16-PAM modulation 


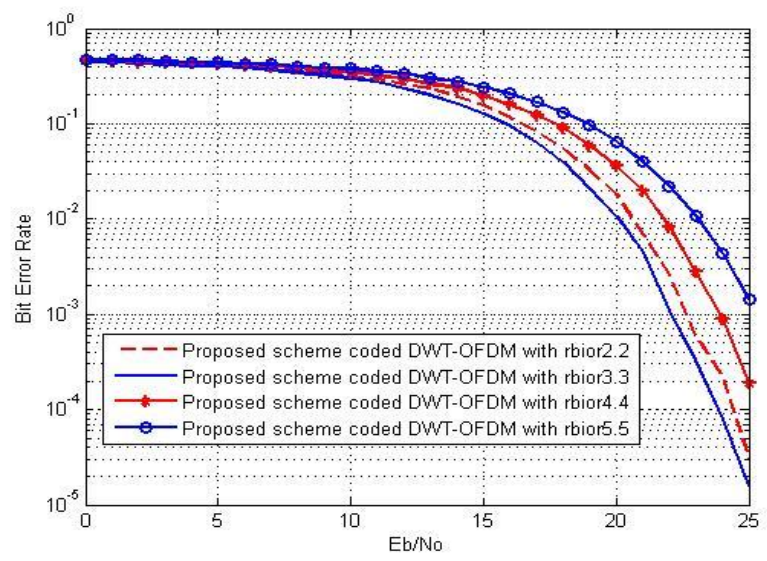

Figure 7 Performance comparison of proposed system using different members of reverse biorthogonal wavelet family

Figure 8 represents the performance of proposed scheme coded DWT-OFDM system with haar, biorthogonal5.5 wavelet and reverse biorthogonal5.5 wavelet using 16-PAM modulation over AWGN channel. Proposed scheme coded DWT-OFDM performs best with biorthogonal5.5 with 16PAM modulation.

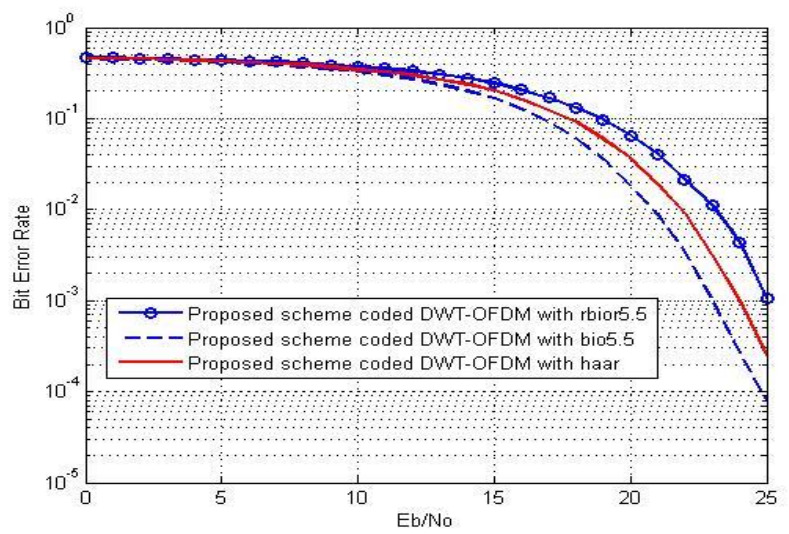

Figure 8 Performance of proposed system with haar, biorthogonal5.5 and reverse biorthogonal5.5 wavelet using 16PAM modulation

\section{CONCLUSION}

Wavelet Transforms is an Important Part of, Systems Theory and Signal Processing and finds numerous important applications in Science and Engineering. In this paper, an efficient error correction is implemented on DWT-OFDM system using 16-PAM modulation scheme over AWGN channel. It is seen from the results that proposed scheme coded DWT-OFDM system outperforms the $1 / 2$ rate convolution encoded DWT-OFDM system with 16-PAM modulation scheme. The performance of the proposed system is studied using different types of wavelets. Haar, biorthogonal and reverse biorthogonal wavelets are used with 16-PAM modulation scheme to simulate the communication system. It is clear from the results that proposed system performs better than convolution coded system with all three types of wavelets. Different members of the same wavelet family are considered to compare their relative performance. Further, it is shown in the results that biorthogonal 5.5 wavelet are best choice for the system. This study finds important applications in Communications and Signal Processing.

\section{ACKNOWLEDGMENT}

Authors are thankful to anonymous reviewers for their careful reading and useful comments for the improvement of the present research work. Also, authors gratefully acknowledge the support provided by the I K Gujral Punjab Technical University, Kapurthala, Punjab, India.

\section{References}

[1] M Engels, Wireless OFDM Systems: How To Make Them Work?, Kluwer Academic Publishers, 2002

[2] G.Ungerboeck, "Channel coding with multilevel phase signals," IEEE Trans. Inform. Theory, vol. 28, pp.55-67, 1982.

[3] Y. Wu and W. Y. Zou, "Orthogonal frequency division multiplexing: a multi-carrier modulation scheme," IEEE Trans. on Consumer Elec., vol. 41, pp. 392-399, 1995.

[4] B. G. Negash and H. Nikookar, "Wavelet-based multicarrier transmission over multipath wireless channels," IEEE Electronics Letters, vol.36, no.21, pp.1787-1788, 2000.

[5] M.J. Manglani and A.E. Bell, "Wavelet Modulation Performance in Gaussian and Rayleigh Fading Channels," In Proc. of MILCOM McLean, Virginia, 2001.

[6] R.W. Hamming, "Error detecting and error correcting code," The bell system tech. journal, vol. 29, no.2, pp.147-160, 1950.

[7] S. Lin, D.J. Costello and M. Miller, "Automatic repeat request error control schemes," IEEE Comm. Magazine, vol.22, no.12, pp. 5-17, 1984.

[8] T. Mizuochi, " Recent progress in forward error correction and its interplay with transmission impairments," IEEE journal on selected topics in quantum electronics, vol. 12, no. 4, 2006.

[9] M.P. Singh and P. Kumar, "An efficient forward error correction scheme for wireless sensor network," Procedia technology, vol.4, pp.737-742, 2012.

[10] K. A. Hasan, M. Waleed and N. Saad, "The performance of multwavelets based OFDM system under different channel conditions," Digital signal Processing, vol. 20, no.2, pp. 472-484, 2010.

[11] M. K Gupta and S. Tiwari, "Performance evaluation of conventional and wavelet based OFDM system," International Journal of Electronics and Comm.," vol. 67, pp.348-354, 2013.

[12]H. Kaur, M. Kumar, A. K. Sharma and H.P. Singh, "Performance analysis of DWT based OFDM over fading environment for mobile Wi-max," Optik, vol. 127, pp. 544-547, 2016. 
[13] V. Kumbusar and O. Kucur, "Performance comparison of wavelet based and conventional OFDM system in multipath Rayleigh fading channels," Digital signal processing, vol. 22, pp.841-846, 2012.

[14]K. Fazel and L. Papke, "On the performance of convolutionally-coded CDMA/OFDM for mobile communication system," In Proc. of IEEE PIMRC, Yokohama, Japan, 1993, 468-472

[15] M.G. Rashed, M.H. Kabir, M.S. Reza, M.M. Islam, R.A. Shams, M. Saleh, S.E. Ullah, "Transmission of Voice Signal: BER Performance Analysis of Different FEC Schemes Based OFDM System over Various Channel," International Journal of Advanced Science and Technology, vol. 3, 2011.

[16] A. Kafaltiya and P.S. Sharma, "Performance improvement in MIMO-OFDM using BCH coding and interleaving," International journal of computer applications, vol. 97, no. 2, 2014.

[17] L. Kansal, V. Sharma and J. Singh, "BER assessment of FEC incorporated OFDM-MPSK wireless system," In Proc. of Fifth International conference on advance computing and communication technologies, India, 21-22 feb, 2015.

[18] N. Hader, M. Bhuyan, M. Ahmed, M. Faisel and M. Salauddin, "Bit Error rate performance analysis of Convolutional coded orthogonal frequency division multiplexing system," In proc. of $57^{\text {th }}$ international scientific conference on power and electrical engineering of Riga technical university, 13-14 october, 2016.

[19]K. Pooja, B.R Rajatha, G.B Sadhvika and B.S Premananda, "Design and performance analysis of FEC schemes in OFDM communication system," In proc. of IEEE international conference on recent trends in electronics, information and communication technology, Benguluru, India, 2016.

[20] V. Sharma and G .Singh, "BER assessment of conventional OFDM and wavelet based OFDM over AWGN channel, Optic, vol. 125, pp. 6071-6073, 2014.

[21] S. Garg, A.K Sharma, A.K Tyagi, "Modified Convolution Coding Scheme for correcting double errors," ITEE Journal Information Technology and Electrical Engineering, Vol. 8, No. 3, pp. 18-24, 2019.

[22] Yong-Song Kim, Tong-Chol Choe, Kum-Hyok Hwang, Method of Generating Intrinsic Mode Functions through a Filtering Algorithm based on Wavelet Packet Decomposition, WSEAS Transactions on Signal Processing, pp. 162-169 Volume 15, 2019
[23] Luqman Hakim, Muhammad Ihsan Zul, Implementation of Discrete Wavelet Transform on Movement Images and Recognition by Artificial Neural Network Algorithm, WSEAS Transactions on Signal Processing, pp. 149-154, Volume 15, 2019

[24] Kim Kyong-Il, Kim Song-Chol, Li Song-Chon, Choe RyuChol, An Improved Thresholding Method for Wavelet Denoising of Acoustic Signal WSEAS Transactions on Signal Processing, pp. 65-76, Volume 15, 2019

[25] Bhavna Harne, A. S. Hiwale, Explore the Effect of Om Mantra Meditation on Brain with Wavelet Analysis, WSEAS Transactions on Signal Processing, pp. 30-38, Volume 15, 2019

\section{Creative Commons Attribution License 4.0 (Attribution 4.0 International, CC BY 4.0)}

This article is published under the terms of the Creative Commons Attribution License 4.0

https://creativecommons.org/licenses/by/4.0/deed.en_US 